\title{
C-R 流形上的叶状结构 *
}

韩祖宏

(上海交通大学应用数学系,上海 200030)

挴要

本文通过对 C-R 流形上的 Pfaffian 系的分析，证明了一般抽象定义的 C-R 流 形上存在 $\mathrm{C}-\mathrm{R}$ 叶及复叶，并给出了这些叶的性质，其中部分关于隻叶的结果对 $C^{n}$ 中 $C-R$ 子流形由 Freeman ${ }^{[1]}$ 所得,而 $\mathrm{C}-\mathrm{R}$ 叶的结果则是新的.

\section{关锤词: C-R 流形，Lovi 形式，可积性，叶状结构}

设 $M$ 为光滑实流形, $g$ 为 $M$ 上复余切子丛, 满足:

1). $\left.g+g=C T^{*} M, 2\right) d g \subseteq \operatorname{ideal}(g)$, 则称 $g$ 为 $M$ 上的一个 $\mathrm{C}-\mathrm{R}$ 结构.

命 $\operatorname{dim}_{c} g=n, \operatorname{dim}_{c} g \cap g=d$, 则 $\operatorname{dim} M=2 n-d$.

有两个特殊情况可以帮助我们理解 $\mathrm{C}-\mathrm{R}$ 结构是介于复结构与实结构之间:

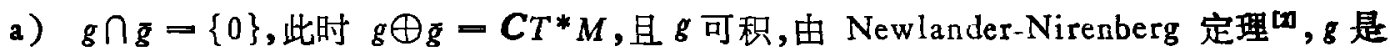
$M$ 上的复结构.

b) $g=g$, 此时 $g=C T^{*} M, g$ 即为 $M$ 上普通的实结构.

$\mathrm{C}-\mathrm{R}$ 流形最典型的例子是 $C *$ 中的实子流形, 即:

$$
M=\left\{z \in C^{*}: \rho_{1}(x)-\cdots-\rho_{d}(x)=0\right\},
$$

其中 $\rho_{1}, \cdots, \rho_{d}$ 为实光滑函数, 且 $\partial \rho_{1} \wedge \cdots \wedge \partial \rho_{d} \neq 0$.

命 $g=\left.s p\left\{d z_{1}, \cdots, d z_{s}\right\}\right|_{u}$, 容易验证 $g$ 即为 $M$ 上的 C-R 结构. $g \cap \bar{g}=\operatorname{sp}\left\{\partial_{\rho_{1}}, \cdots, \partial \rho_{d}\right\}$, Freeman ${ }^{\text {Id }}$ 讨论了这种 $C$ * 中的 C-R 流形上的 Levi 复叶.

本文考虑的对象是抽象定义的 C-R 流形,命 $g \cap \bar{g}-g_{0} \otimes_{R} C$, 则 $g_{0}$ 是 $M$ 上 $d$ 维实分布.

\section{一、Levi 形式和 $\boldsymbol{g}_{0}$ 的可积性}

$(M, g)$ 为前面定义的 C-R 流形, $g \cap g=g_{0} \otimes_{R} C, \Lambda^{p}(M)$ 表示 $M$ 上 $p$ 形式, $d: \Lambda^{p}(M) \rightarrow$ $A^{p+1}(M)$ 是外微分算子, 作映射:

$$
\delta_{1}: g_{0} \rightarrow \Lambda^{2}(M) / \Lambda^{2}(M) \wedge g_{0},
$$

$\delta_{1}=\pi^{\circ} \circ, \pi: \Lambda^{2}(M) \rightarrow \Lambda^{2}(M) / \Lambda^{1}(M) \wedge g_{0}$ 是投影. 显然 $\delta_{1}$ 是 $C(M)$ 同态, 命 $\delta_{1}-$ ker $\delta_{1}$, 则有正合序列: 


$$
0 \rightarrow g_{1} \stackrel{i}{\rightarrow} g_{0} \stackrel{\delta_{1}}{\rightarrow} \delta_{1}\left(g_{0}\right) \rightarrow 0,
$$

其中 $\delta_{1}\left(g_{0}\right)-\pi^{\circ} d\left(g_{0}\right)=d g_{0} \bmod \left(g_{0}\right)$.

归纳地可以定义映射:

$$
\delta_{r}: g_{r-1} \rightarrow \delta_{r}\left(g_{r-1}\right)=d g_{r-1} \bmod \left(g_{r-1}\right),
$$

命 $g_{r}=\operatorname{ker} \delta_{r}$, 则有正合序列:

$$
0 \rightarrow g_{r} \stackrel{i}{\rightarrow} g_{r-1} \stackrel{\delta_{r}}{\rightarrow} \delta_{r}\left(g_{r-1}\right) \rightarrow 0,
$$

称 $g_{1}, g_{2}, \cdots, g_{r} \cdots$ 是 $g_{0}$ 的导出系, 如果 $g_{0}$ 的所有导出系都是常秩, 称 C-R 流形是正则的, 本文总假设 C-R 流形是正则的.

$l$-Levi 形式定义为胦射: $\delta_{l}: g_{l-1} \rightarrow \delta_{l}\left(g_{l-1}\right)$.

如果 $l$-Levi 形式恒为零, 即 $\delta_{l} \equiv 0$, 则 $d g_{l-1} \equiv 0 \bmod \left(g_{l-1}\right)$, 即 $g_{l-1}$ 是可积的, 而且 $g_{l-1}=$ $g_{l}=g_{l+1}=\cdots$,

因此,Levi 形式度量了全实子丛 $g_{0}$ 的可积性.

令 $g_{\mathrm{int}}=\bigcap_{r>0} g_{r}$, 由于 $g_{r} \supseteq g_{r+1}$, 目 $M$ 正则, 则必存在最小正整数 $N$ 使得: $g_{p+N}=g_{N} \forall p$ $\geqslant 0$, 从而 $g_{\mathrm{int}}-g_{N}$, 剙且 $\delta_{N+1} \equiv 0$, 从而 $g_{\mathrm{in} z}$ 是可积的. 由Frobenius定理, 存在 $M$ 上的叶状 结构 $\mathscr{E}$, 使得 $\mathscr{E}$ 中的每个叶 $N$ 都是 $g_{\mathrm{in}_{\mathrm{n}}}$ 的积分子流形, 并且 $\operatorname{dim} N-2 n-d-(d-e)=$ $2 n-2 d+e$,

其中 $e=\mathrm{dim} g_{0} / g_{\text {in }}$ 为 Levi 代数余维.

下面讨论 $\mathscr{E}$ 中叶的性质, $N \in \mathscr{E}$,

令 $g^{*}-g \cap C T^{*} N$, 则 $g^{*}$ 为 $C T^{*} N$ 的复子丛, 而且

$$
g^{*}+\bar{g}^{*}-(g+\xi) \cap C T^{*} N=C T^{*} N,
$$

因为 $d g^{*} \equiv 0 \bmod g \equiv 0 \bmod C T^{*} N$,

所以 $d g^{*} \equiv 0 \bmod g \cap C T^{*} N \equiv \operatorname{0mod}\left(g^{*}\right)$.

这说明 $g^{*}$ 为 $N$ 上的 C-R 结构,

$\left(g^{*}\right)^{\perp} \cap C T N=g^{\perp}$, 故 $\left(N, g^{*}\right)$ 与 $(M, g)$ 具有相同的复切空间.

令 $g_{0}^{*} \otimes_{R} C-g^{*} \cap g^{*}$, 同上, 令 $\left\{g_{r}^{*}\right\}$ 为 $g_{0}^{*}$ 的导出系, 由于

$$
\begin{aligned}
\bar{g}^{*} \cap g^{*} & =\left(g \cap C T^{*} N\right) \cap\left(g \cap C T^{*} N\right) \\
& =(g \cap g) \cap C T^{*} N=\left(g_{0} \cap T^{*} N\right) \otimes_{k} C
\end{aligned}
$$

可知: $g_{0}^{*}-g_{0} \cap T^{*} N$.

由归纳法容易看出, $g_{r}^{*}=g_{r} \cap T^{*} N$,

因此, $g_{\mathrm{int}}^{*}-\bigcap_{r>0} g_{r}^{*}-g_{\mathrm{in}_{\mathrm{a}}} \cap T^{*} N=\{0\}$.

对 C-R 流形 $(M, g)$,如果 $g_{\mathrm{int}}-\{0\}$,称 $(M, g)$ 是完全不可积的,如果 $g_{\mathrm{iat}}-\mathrm{s}_{0}$, 称 $(M, g)$ 为完全可积.

综上所述,我们有以下结论:

定理 1.1. $(M, g)$ 上存在叶状结构 $\mathscr{E}$, 使得 $\mathscr{E}$ 中的叶都是 $M$ 的完全不可积 C-R 子流 形,并且和 $M$ 具有相闰的复切空间。 
有两个特殊情况:

a) $g_{\mathrm{int}}=\{0\}$, 此时 $e-d$, 从而 $N=M$, 即除 $M$ 本身以外, 不存在 $M$ 的子 $\mathrm{C}-\mathrm{R}$ 流形与 $M$ 具有相同的复切空间,并且是完全不可积的.

b) $g_{\mathrm{int}}=g_{0}$, 此时 $e=0$, 由复 Frobenius 定理 ${ }^{[3]}$, 定理 2.1 中的叶是 $\mathbf{n}-\mathbf{d}$ 维复叶.

Pfäffian 系 $g_{\text {int }}$ 的维数在 C-R 函数的扩充中起很重要的作用, 如果, $M$ 为 $C$ 中的 C$\mathrm{R}$ 子流形, 由文献 [4] 中的结果, $M$ 上的 $\mathrm{C}-\mathrm{R}$ 函数可延拓到另一 C-R 子流形 $\widetilde{M}$ 上的 CR 函数,其中 $\operatorname{dim} \tilde{M}-a n-d+e$, 因此当 $g_{0}$ 完全不可积时, $M$ 上的 C-R 函数可全纯延拓 到 $C$ 中某开集上, 而当 $g_{0}$ 完全可积时, 由文献 [5]， $M$ 是全纯凸的, 即 C-R 函数不能作任 何扩充, 此时 $\widetilde{M}=M$. 另一方面, 由于定理 1.1 中的 C-R 叶与 $M$ 具有相同的复切空间, 因 此, $M$ 上的 C-R 函数限制在每个叶上也都是 C-R 函数, $M$ 上的 C-R 函数的全纯扩充即可 化为每个叶上 C-R 函数的扩充问题.

下面给出 $g_{0}$ 的可积性的几何意义, 取 $g_{0}$ 的局部基 $\left\{\theta^{1}, \theta^{2}, \cdots, \theta^{d}\right\}$, 将其扩充为 8 的局 部基 $\theta^{1}, \cdots, \theta^{d}, \omega^{1}, \cdots, \omega^{*-\alpha}$ 由于 $d g \equiv \operatorname{0mod}(g)$,及 $\theta^{j}, 1 \leqslant j \leqslant d$ 是实形式,则有

$$
d \theta^{i}=i g_{\alpha \beta}^{j} \omega^{\alpha} \wedge \omega^{\beta} \bmod \theta^{k}, 1 \leqslant j, k \leqslant d, 1 \leqslant \alpha, \beta \leqslant n-d .
$$

$\left(g_{a \beta}^{i}\right)$ 为 Hermitian 方阵, 不妨设 $g_{\mathrm{ist}}=\operatorname{sp}\left\{\theta^{1}, \cdots, \theta^{d-e}\right\}$,

则 $d \theta^{s} \equiv 0 \bmod \theta^{r}, 1 \leqslant s, r \leqslant d-e$,

从而有 $\left(g_{\alpha \beta}^{j}\right) \equiv 0,1 \leqslant i \leqslant d-e$,因此:

性质 1.1. 1) $g_{0}$ 完全可积 $\Leftrightarrow\left(g_{a \beta}^{i}\right)=0(1 \leqslant j \leqslant d-e)$. 2) $g_{0}$ 完全不可积 $\Leftrightarrow\left(g_{a \beta}^{i}\right) \neq 0$, $1 \leqslant j \leqslant d$.

\section{二、Levi 复 叶}

本节研究 C-R 流形中的复叶状结构. 对 $C$ 中的 C-R 流形, 定理 2.1 由 Freeman ${ }^{\text {W }}$ 所 得, 本文给出的方法适用抽象定义的 C-R 流形, 直接利用 C-R 结构给出 Levi 分布, 如果 将这些限制在 $\boldsymbol{C}^{\boldsymbol{n}}$ 中的 C-R 子流形将得到 Freeman ${ }^{[\mathrm{d}}$ 的结果.

设 $N \stackrel{i}{\rightarrow} M$ 是全纯浸人,且 $i^{*} g_{0}=0$, 则称 $N$ 为 $M$ 的可容复子流形,取 $g_{0}$ 的局部基 $\left\{\theta^{1}, \cdots\right.$, $\left.\theta^{d}\right\}$, 则有 $i^{*} \theta^{j}-0$, 两边微分得到: $i^{*}\left(i g_{a \beta}^{j} \omega^{\alpha} \wedge \bar{\omega}^{\beta}\right)=0$.

由此可知，如果对某 $j,\left(g_{a \beta}^{\prime}\right)$ 是正(负)定, 则 $M$ 上不存在可容复子流形,特别，如果 $M$ 是严格 拟凸的,则 $M$ 上不存在复子流形。

命: $\left.L M-\left\{X \in g_{0}^{\frac{1}{0}}: d g_{0}\right\rfloor X=0\right\}, L M$ 称为 Levi 分布, 则有:

定理 2.1. $M$ 上存在由复流形组成的叶状结构 $\mathscr{L}$, 使得 $T \mathscr{L}=L M$, 这里 $T \mathscr{L}$ 表示 $\mathscr{L}$ 中叶的切空间.

证. 令: $\left.L^{1.0}-\left\{Z \in \bar{g}^{\perp}, d g_{0}\right\rfloor Z=0\right\}$,

首先证明 $L^{1.0}$ 在 Frobenius 意义下是可积的,因为 $d g \equiv 0 \bmod (g)$, 因此, 对 $Z_{1}, Z_{1} \in g^{\perp}$. 有 $\left[Z_{1}, Z_{2}\right] \in \bar{g}^{\perp}$. 设 $Z_{1}, Z_{2} \in L^{1.0}$, 则由

$$
\left.\left.\left.d g_{0}\right\rfloor\left[Z_{1} Z_{2}\right]-\mathscr{L}_{Z_{1}}\left(d g_{0}\right\lrcorner Z_{2}\right)-\left(\mathscr{L}_{Z_{1}} d g_{0}\right)\right\rfloor Z_{2},
$$

其中 $\mathscr{L}_{Z}$ 表示关于 $Z$ 的 Lie 导数.

由于 $\left.\left.d g_{0}\right\lrcorner Z_{1}=0, d g_{0}\right\lrcorner Z_{2}=0$, 则

$$
\left.\left.\mathscr{L}_{Z_{1}} d g_{0}=d\left(d g_{0}\right\lrcorner Z_{1}\right)+\left(d \circ d g_{0}\right)\right\lrcorner Z_{1}-0,
$$


所以 $\left.d g_{0}\right\rfloor\left[Z_{1}, Z_{2}\right]-0$, 即 $\left[Z_{1}, Z_{2}\right] \in L^{1.0}$.

由于 $g_{0} \otimes_{R} C-g \cap_{g}$, 从而 $\left(g_{0}^{\frac{1}{0}}\right) \otimes_{R} C-g^{\perp} \oplus_{g^{\perp}}$, 故:

$$
L M \otimes_{R} C=L^{1.0} \oplus \overline{L^{1.0}} \text {. }
$$

下面来证明 $L M \otimes_{R} C$ 是可积的. 设 $X, Y \in L M$, 完全类似上面的证明可知: $\left.d g_{0}\right\rfloor[X, Y]=$ 0 , 剩下只要说明 $[X, Y] \in g_{0}^{\perp}$, 由下列公式,

$$
\left.\left.\left.g_{0}\right\rfloor[X, Y]-\mathscr{L}_{X}\left(g_{0}\right\lrcorner Y\right)-\left(\mathscr{L}_{\times} g_{0}\right)\right\rfloor Y,
$$

而 $\left.\left.\mathscr{L}_{x} g_{0}=d\left(g_{0}\right\rfloor X\right)+d g_{0}\right\rfloor X$. 由于 $\left.\left.\left.g_{0}\right\rfloor X=g_{0}\right\rfloor Y=0, d g_{0}\right\rfloor X=0$, 可知 $\left.g_{0}\right\rfloor$ $[X, Y]-0$, 即 $[X, Y] \in g_{0}^{\frac{1}{0}}$.

我们假定 $M$ 是正则, 即 $L M$ 是常秩,因此由复 Frobenius 定理, 定理得证.

注 1. 如果 $M-\left\{z \in C^{*}: \rho_{1}-\cdots-\rho_{d}=0\right\}$ 为 $C^{*}$ 中的 C-R 子 流形, 命 $d^{*}-$ $-i(\partial-\bar{\partial})$, 则 $g_{0}-\operatorname{sp}\left\{d^{c} \rho_{1}, \cdots, d^{c} \rho_{d}\right\}$,

$$
\begin{gathered}
\left.L M-\left\{X \in g_{0}^{\perp}: d d^{*} \rho_{i}\right\lrcorner X=0,1 \leqslant i \leqslant d\right\} \\
\left.-\bigcap_{i=1}^{b}\left\{X \in g_{0}^{\perp}: \partial \partial_{\rho_{j}}\right\rfloor X-0\right\},
\end{gathered}
$$

因此,定理 2.1 限制到 C* 中 C-R 流形时,与文献 [1] 中的结果相一致.

注 2. 当 $L M=\{0\}$ 时, $M$ 上的复叶退化成点, 而当 $L M=g_{0}^{\perp}$ 时, 则 $d g_{0} \equiv 0 \bmod g_{0}$ ， 即 $g_{0}$ 是完全可积的, 此时复叶为 C-R 叶,而当 Levi 叶为 C-R 叶时, $g_{0}$ 则完全可积.

定理 2.1 所确定的叶称为 Levi 复叶,下面来研究 Levi 复叶的几何性质.

取 $g_{0}$ 的一组基 $\theta^{1}, \cdots, \theta^{d}$, 将其扩充成为 $C T^{*} M$ 的一组正交基 $\omega^{1}, \cdots, \omega^{n-d}, \omega^{1}, \cdots$, $\omega^{*-d} \theta^{1}, \cdots, \theta^{d}$, 命 $Z_{1}, \cdots, Z_{*-d}$ 为对偶于 $\omega^{1}, \cdots, \omega^{*-d}$ 的向量场, 则 $Z_{1}, \cdots, Z_{n-d}$ 局部张 成 $M$ 的全纯切空间.

命 $d \theta^{i}-i g_{\alpha \beta}^{i} \omega^{a} \wedge \omega^{\beta} \bmod \theta^{k}$,

则 $\delta_{1} \theta^{i}-i g_{\alpha \beta}^{i} \omega^{\alpha} \wedge \omega^{\beta}$.

对 $Z-a^{a} Z_{a} \in L^{1.0}$, 则由 $d g_{0} \equiv \delta_{1} g_{0} \bmod g_{0}$,

$$
\left.\left.0=d g_{0}\right\rfloor Z-\delta_{1} g_{0}\right\rfloor Z \bmod g_{0}
$$

对任何 $W-b^{\beta} Z_{\beta} \in \tilde{g}^{\perp}$, 与上式缩并，

即

$$
\begin{gathered}
\left.\left.\delta_{1} g_{0}\right\rfloor Z\right\lrcorner W-0, \\
g_{\alpha \beta}^{j} a^{a} b^{\beta}-0 \quad 1 \leqslant j \leqslant d .
\end{gathered}
$$

由W任意性,可知 $g_{a \beta}^{i} a^{\alpha}-0$, 这说明 $Z \in L^{1.0}$ 是每个 $\left(g_{\alpha \beta}^{i}\right)$ 的左䗙特征向量场, 很容易 验证这些左零特征向量场是不依赖于 $g_{0}$ 的局部基的选择, 而仅依赖 $M$ 上的 C-R 结构. 根据 以上分析有显然的推论.

推论 2.1. 1) 如果存在某个 $\left(g_{\alpha \beta}^{\prime}\right)$ 非异, 则 $M$ 上不存在 Levi 复叶, 2) 如果 $M=$ $\left\{z \in C^{*}: \rho(z)=0\right\}$ 为 $C^{*}$ 中实超曲面, 则 $M$ 上存在 $m$ 维 Levi 复叶, 当且仅当 $M$ 的 Levi 形 式 $\partial \partial_{\rho}$ 具有 $m$ 个零特征值.

一个自然的问题是: Levi 复叶是否包含了所有的复叶? Sommer ${ }^{[6]}$ 给出以下例子:

$$
M=\left\{z \in C^{3}: \rho-\left|z_{1}\right|^{2}+\left|z_{2}\right|^{2}-\left|z_{3}\right|^{2}-1=0\right\},
$$

易知 $L M-\{0\}, M$ 上无 Levi 复叶,但有下面 1 参数复叶: 


$$
N(\theta)-\left\{\left(z_{1}, z_{2}, 0\right)+\tau\left(-\bar{z}_{2}, \bar{z}_{1}, e^{i \theta}\right),\left|z_{1}\right|^{2}+\left|z_{2}\right|^{2}-1, \tau \in C\right\} .
$$

对非 Levi 复叶,Bryant ${ }^{\text {m }}$ 对 Lorentzian C-R 流形上的全纯曲线做了深入的研究,Abe 给出 C 中 C-R 流形上复叶(非 Levi) 的一个充分必要条件,但这个条件非常复杂,而且似 乎与 C-R 结构并无联系, 通过 C-R 结构直接刻画出非 Levi 复叶的分布至今尚未见到.

下面来说明在一定情况下,Levi 叶是极大的.

一般地,设 $N \subset M$ 是可容复子流形,设 $X \in T^{1.0} N$, 则有 $\left.g_{0}\right\rfloor X-0$, 两边微分,

$$
\left.\left.0-d\left(g_{0}\right\lrcorner X\right)=\mathscr{L}_{x g_{0}}-d g_{0}\right\rfloor X,
$$

即

$$
\left.d g_{0}\right\lrcorner X-\mathscr{L}_{\mathrm{X} g_{0}} \text {, }
$$

两边与 $\bar{X}$ 缩并，

$$
\begin{aligned}
\left.\left.d g_{0}\right\rfloor X\right\rfloor \bar{X} & \left.=\mathscr{L}_{X} g_{0}\right\rfloor \bar{X} \\
& \left.=\mathscr{L}_{X}\left(g_{0} \Gamma \bar{X}\right)-g_{0}\right\rfloor[X, \bar{X}]=0 .
\end{aligned}
$$

如果令 $\left.\left.Z^{1.0}=\left\{Z \in \bar{g}^{\perp}: d g_{0}\right\rfloor Z\right\rfloor \bar{Z}-0\right\}$,

则对任何可容复子流形 $N$, 都有 $T^{1.0} N \subseteq Z^{1.0}$.

注意 $Z^{1.0}$ 不是线性空间,一般有关系:

$$
L^{1.0} \subset Z^{1.0} \subset \bar{g}^{1} \text {. }
$$

由于下面的定理,当 $L^{1.0}-Z^{1.0}$ 时, 称 $M$ 是半定的.

定玨 2.2. 如果 $\operatorname{dim} g_{0}=1$, 则 $L^{1.0}-Z^{1.0}$ 当且仅当 $M$ 的 Levi 形式是半定的.

证. $\theta \in g_{0}, \omega^{1}, \cdots, \omega^{-1}, \theta$ 为 $g$ 的一组基, 则

$$
d \theta-i g_{{ }_{s} \bar{\beta}} \omega^{\alpha} \wedge \bar{\omega}^{\beta} \bmod \theta \text {, }
$$

从而 $L^{1.0}-\left\{a \in \boldsymbol{C}^{=-1}, g_{\alpha \beta} a^{\alpha}-0\right\}$,

$$
Z^{1.0}-\left\{a \in C^{n-1}: g_{a \beta} a^{\alpha} a^{\beta}-0\right\} .
$$

设 $G=\left(g_{\alpha \beta}\right)$, 如果 $G$ 是半定, 则存在方阵 $F$, 使得 $G-P \cdot \bar{P}^{r}$, 如果 $a \in Z^{1.0}$, 则 $a \bar{P}^{\prime} \bar{a}^{\prime}=$ 0 ,

即 $(a P) \overline{(a P)^{\prime}}=0, a \dot{P}-0$, 从而 $a G=a P \cdot \bar{P}^{\prime}=0, a \in L^{1.0}$, 故 $L^{1.0}-Z^{1.0}$ 。

汉之,若 $L^{1.0}-Z^{1.0}$, 设 $\left(g_{a B}\right)$ 不是半定的,不失一般性,设

$$
\left(g_{\alpha \beta}\right)=\left(\begin{array}{ccc}
\lambda_{1}, & & \\
\lambda_{2}, & & 0 \\
0 & \ddots & \\
\lambda_{=-1}
\end{array}\right) \text { 为对角形, }
$$

且 $\lambda_{1}>0, \lambda_{2}<0$, 取 $a-\left(\frac{t}{\sqrt{\lambda_{1}}}, \frac{t}{\sqrt{-\lambda_{1}}}, 0,0, \cdots, 0,\right) t \in C$.

则 $a \in Z^{1.0}$, 但 $a \notin L^{1.0}$. 证毕.

从上面的讨论可知, 当 $M$ 是半定时, Levi 复叶是极大的.

最后给出 Levi 复叶的一个特征:

定理 2.3. 设 $\mathscr{L}$ 表示 $M$ 上的 Levi 复叶,则对 $\mathscr{L}$ 中的每个叶 $L \in \mathscr{L}, g \cap \bar{g}$ 为 $L$ 上的 全纯向量丛,亦称 $g \cap g$ 为 $\mathscr{L}$ 上的全纯向量丛. 如果 $\mathscr{M}$ 为 $M$ 上任一可容复叶, 满足 $g \cap \mathrm{I}$ 为 $\mathscr{M}$ 上的全纯向量丛, 则

$$
T^{1.0} \mathscr{M} \subseteq T^{1 . \nu} \mathscr{L}
$$

$T^{1.0}$ 表示叶的全纯切向量. 
证. $L \in \mathscr{L}, U \subset L$ 为 $L$ 的开子集， $\eta_{1}, \cdots, \eta_{d}$ 为 $U$ 上 $g \cap \bar{g}$ 的复基， $V \subset L$ 为另一开 集, $\zeta_{1}, \cdots, \zeta_{d}$ 为 $V$ 上 $g \cap g$ 的复基, 则存在 $U \cap V$ 上的非异方阵 $\left(a_{i k}\right)$ 使得在 $U \cap V$ 上有:

$$
\begin{aligned}
\zeta_{j} & =\sum_{k} a_{i k} \eta_{k}, \\
d \zeta_{j} & =\sum_{k} a_{j k} d \eta_{k}+\sum_{k} d a_{j k} \wedge \eta_{k} .
\end{aligned}
$$

壬取 $X \in L^{0.1}$, 则

$$
\left.\left.0=d \zeta_{i}\right\lrcorner X-\sum_{k}\left(d a_{i k} \wedge \eta_{k}\right)\right\lrcorner X-\sum_{k} X\left(a_{i k}\right) \eta_{k},
$$

即 $X\left(a_{i k}\right)-0, \partial_{L} a_{i k}-0$, 故 $g \cap g$ 为 $L$ 上的全纯向量丛.

若 $g \cap g$ 为 $\mathscr{A}$ 上的全纯向量丛，因此局部存在方阵 $\left(C_{i k}\right)$ 使得 $\sum_{k} C_{i k} \eta_{k}, 1 \leqslant j \leqslant d$ 为 $g \cap g \mid$ 上的全纯基,其中 $\eta_{1} \cdots, \eta_{d}$ 为 $\mathscr{M}$ 上 $g \cap \tilde{g}$ 的复基.

则

$$
\begin{gathered}
\partial_{-}\left(\sum_{k} C_{i k} \eta_{k}\right)-0, \text { 即 } \\
\sum_{k} \partial_{\curvearrowright} C_{i k} \wedge \eta_{k}+\sum_{k} C_{i k} \partial_{\propto} \eta_{k}=0 .
\end{gathered}
$$

对 $z \in T^{1.0} \mathscr{A}$,与上式缩并,由 $\left(C_{i k_{k}}\right)$ 可逆,知:

$$
\left.\partial_{\wedge}\right\lrcorner Z-0 \quad 1 \leqslant k \leqslant d,
$$

如果 $Z \in T^{1.0} \mathscr{L}$, 存在 $k$, 使得

$\left.d \eta_{k}\right\lrcorner Z \neq 0$, 限制在 $\mathscr{C}$ 上: $\left.\bar{\partial}_{\propto} \eta_{k}\right\lrcorner Z \neq 0$.

从而产生矛盾! 命题得证.

注. 对非 Levi 复叶上述不成立,例如 Sommer 例子:

$$
\begin{gathered}
M-\left\{z \in C^{3}: \rho=\left|z_{1}\right|^{2}+\left|z_{2}\right|^{2}-\left|z_{3}\right|^{2}-1-0\right\}, \\
\mathscr{N}(\theta)-\left\{\left(z_{1}, z_{2}, 0\right)+\tau\left(-\bar{z}_{1}, \bar{z}_{1}, e^{i \theta}\right): \tau \in C,\left|z_{1}\right|^{2}+\left|z_{2}\right|^{2}-1\right\}, \\
g \cap \bar{g}-\operatorname{sp}\left\{\partial_{\rho}\right\}, \text { 易知 } \partial_{\rho} \text { 在叶 } N(\theta) \text { 上不全纯. }
\end{gathered}
$$

\section{考文城}

[1] Freeman, M, Proc. A. M. S, 57(1976), 369-370.

[ 2] Newander, A. \& Nirenberg. L., Ann. Math, 65(1957), 391-401.

[3] Nirenberg, L., Semirars on enalytic function, I, Princeton, 1957, 172-189.

[4] Hunt, L. R. \& Wells, Jr. R. O, Amer. J. of Mask, 98(1976), 805-820.

[ 5 ] Wells, Jr. R. O., Trans. A. M. S, 132(1968), 245-262.

[6] Sommer, F, Math. Ann, 136(1958), 111-133.

[7] Bryant, R., Trons. A. M. S, 272(1982), 203-221.

[1] Abe, Y, Memoirs of the Foculty of Science, Kywth University Ser. A, 37(1983), 2: 99-108. 